\title{
Assessment of the Contributions of Private Provider Engagement in Tuberculosis Case Finding and Notification in South West Nigeria
}

\author{
Muse 0. Fadeyi ${ }^{*}$, Bolaji Ahmed ${ }^{1}$, Akinwumi Akindele1, Adebayo Bakare ${ }^{1}$, Modupe Ariyo ${ }^{1}$, \\ Olalekan Onitiju' ${ }^{1}$, Osman El-Tayeb ${ }^{1}$, Taofeekat Ali ${ }^{2}$, Yasir Othman ${ }^{3}$, Aderonke Agbaje ${ }^{2}$ \\ ${ }^{1}$ Damien Foundation Belgium (Nigeria Project), Ibadan, Oyo State, Nigeria \\ ${ }^{2}$ Institute of Human Virology Nigeria (IHVN), Abuja, Nigeria \\ ${ }^{3}$ Hull University Teaching Hospitals NHS Trust, Hull, United Kingdom \\ Email: *fadeyi.muse@dfbnigeria.org.ng
}

How to cite this paper: Fadeyi, M.O., Ahmed, B., Akindele, A., Bakare, A., Ariyo, M., Onitiju, O., El-Tayeb, O., Ali, T., Othman, Y. and Agbaje, A. (2021) Assessment of the Contributions of Private Provider Engagement in Tuberculosis Case Finding and Notification in South West Nigeria. Journal of Tuberculosis Research, 9, 160-171. https://doi.org/10.4236/jtr.2021.93015

Received: July 6, 2021

Accepted: August 9, 2021

Published: August 12, 2021

Copyright $\odot 2021$ by author(s) and Scientific Research Publishing Inc. This work is licensed under the Creative Commons Attribution International License (CC BY 4.0).

http://creativecommons.org/licenses/by/4.0/ (c) (i) Open Access

\begin{abstract}
Introduction: Tuberculosis is one of the leading causes of mortalities attributable to an infectious disease. In a private sector-driven health system such as Nigeria, the private health providers are very important stakeholders in tuberculosis diagnosis and management. Unfortunately, there are few data on the level of contribution of these private health providers to the case finding and notification of tuberculosis in Nigeria. Consequently, this study assessed the contribution of the private providers to the diagnosis and treatment of tuberculosis under the Global Fund Private Public Mix New Funding Model ( $2^{\text {nd }}$ phase) grant (GF PPM NFM2) in the four implementing states of south west Nigeria. Materials and Methods: This is a retrospective, cross-sectional analysis of the NFM2 programme implementation data collected between January 1, 2019 and December 31, 2020. Data was extracted from the routine registers such as the presumptive TB registers; treatment register of the private health facilities engaged for PPM activities between 2019 and 2020. The data was entered and analyzed using Microsoft excel package. The variables were summarized using appropriate charts and table. Results: Concerning the percentage contribution of the Global Fund Private Public Mix (GF PPM) grant to the total state $\mathrm{TB}$ case notification, majority of the implementing states show some progressive rise in the contribution of GF PPM to State TB case notification as the grant implementation progressed. Furthermore, at the initial period of the grant implementation, the health facilities seemed to have generated majority of the cases. However, as the grant implementation progressed, the PPMVs were responsible for the diagnosis of majority of the cases. Conclusion: As the grant progressed, there was a progressive increase in the num-
\end{abstract}


ber of presumptive TB cases as well as in the number of notified cases of tuberculosis attributable to private provider engagement.

\section{Keywords}

Private Provider, Tuberculosis, Diagnosis, Notification

\section{Introduction}

Tuberculosis (TB) remains one of the biggest public health problems affecting mankind. It is caused by Mycobacterium tuberculosis. It typically affects the lungs but it can also affect other organs such as the bone; brain; meninges; and kidneys [1]. Worldwide, it has been adjudged as the leading cause of death from an infectious disease [1]. Globally, about 10 million persons were affected by the disease in 2019 and causing about 1.2 million deaths among HIV negative persons [1]. Though the above figures might reflect a reduction in the global incidence of tuberculosis, this reduction is not occurring at such a level as to attain the 2025 milestone for the global end TB strategy [2]. Furthermore, Nigeria is one of the eight countries which account for two-thirds of the global TB burden [1]. In Nigeria, it has been estimated that over 3 million out of the 10 million cases of tuberculosis are missed, thereby contributing hugely to risk of onward transmission of this disease in the community [1]. Moreover, Nigeria is one of the three countries which account for about $46 \%$ of these missing cases of tuberculosis and it contributes $11 \%$ of the total missed cases worldwide [1].

In developing countries such as Nigeria, private healthcare providers are very important stakeholders in the health systems of these countries [3]. In Nigeria, the private health sector contributes as high as $70 \%$ to the health system delivery of the nation [4]. Moreover, $66 \%$ - $92 \%$ of all cases of respiratory pathologies and fever are reported to be first seen by the private health providers [5]. Evidence has also shown that the private health sector is the first point-of-call in the event of symptoms suggestive of tuberculosis [6] [7]. Thus, there are lots of missed opportunities with respect to tuberculosis case finding in the private health sectors [3].

Consequently, the global attention shifted to the Private-Public Mix (PPM) strategy in the countries with very large burden of missing cases of tuberculosis. In order to increase the private sector contribution to tuberculosis case finding in Nigeria, the second phase of the New Funding Model (NFM2) was funded by the Global Fund (GF) between 2019 and 2020.

At present, there is paucity of data on the level of contribution of the private sector to health care delivery in Nigeria [5]. And where present, such data may be unreliable [5]. Hence, there is the need for more reliable data on the private sector contribution to the treatment and notification of tuberculosis in Nigeria. In the light of the above, this study aims to evaluate the contribution of the pri- 
vate health providers to the tuberculosis case finding and notification under the GF PPM NFM2 grant in the four implementing states in South West Nigeria between 2019 and 2020.

\section{Materials and Methods}

\subsection{Study Setting and Context}

\subsubsection{Study Design}

This is a descriptive analysis of the NFM2 programme implementation data collected between January 1, 2019 and December 31, 2020, to evaluate the contribution of the private healthcare providers to tuberculosis case detection in the selected states in south west Nigeria.

\subsubsection{The NFM2 Programme Implementation}

The Global Fund to Fight AIDS Tuberculosis and Malaria (GFATM) supported the second phase of the New Funding Model (NFM2), which focused primarily on private health providers' engagement, between 2019 and 2020. Several implementation strategies adopted during this cycle included: PPM stakeholder engagement; facility Outpatient Department (OPD) screening strategy; Patient linkage and retention; Free chest X-ray for childhood TB; Service providers incentives and enablers.

In the NFM2 funding cycle, the Damien Foundation Belgium, Nigerian project (DFB) was the sub-recipient to the Institute of Human Virology, Nigeria (IHVN). The implementing states were Oyo; Osun; Ogun and Ondo states. A map of Nigeria showing the four implementing states covered by DFB is as shown in Figure 1 below. The private facilities in these implementing states were supported by DFB through capacity building; mentoring and supportive supervision; as well as onsite data verification. The engagement of the private health providers was preceded by facility assessment by the Local Government TB Supervisors (LGTBLS) in all the districts within the four States. These assessed facilities were then mapped in clusters using the hub and spoke model. In this arrangement, the spokes include: Patent and Proprietary Medicine Vendors (PP$\mathrm{MV}$ ); Community Pharmacists (CPs); the stand-alone laboratories (SAL) as well as Traditional Birth Attendants (TBAs); they screen clients for TB and refer presumptive cases to the hub for further care. The hubs include the Private-for profit (PFP) health facilities and Faith-Based Organizations (FBOs) and provide the full cascade of TB services: screening, diagnosis, treatment and follow-up monitoring.

The spokes were then trained and engaged on Identification \& referral of presumptive TB cases to their respective hubs. The hubs were in turn trained and engaged on Identification of presumptive TB cases, diagnosis of the presumptive TB patients as well as the treatment of the diagnosed TB cases. The services of the hubs as well as those of the spokes were incentivized. Linkage coordinators were engaged to reduce cascade leakages and promote patient retention. Facility assessment, mapping, training, facility signing of Memorandum 


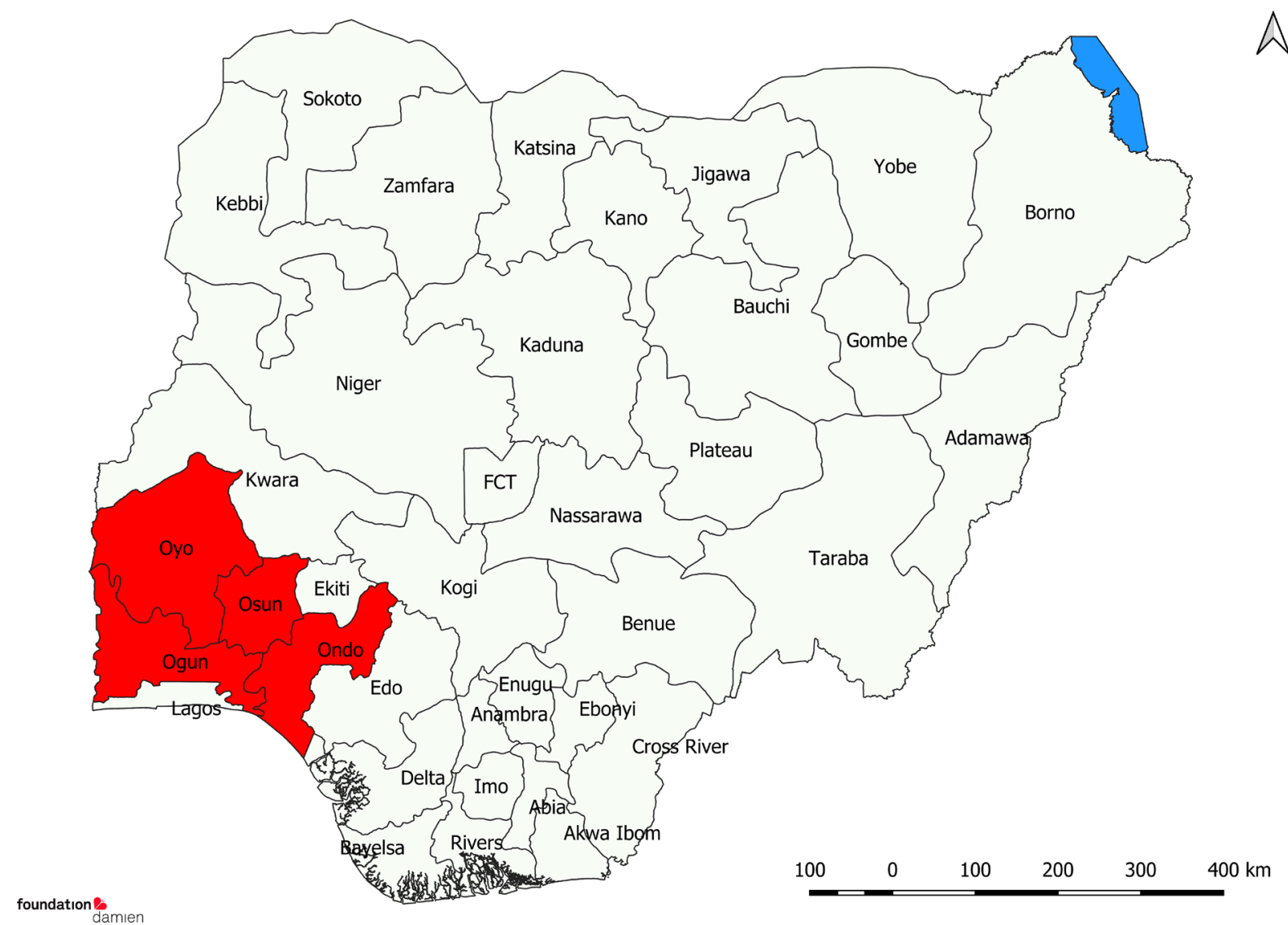

Figure 1. Map of Nigeria showing all the four implementing states covered by DFB in the NFM2 grant.

of understanding (M.O.U) and field workers engagement occurred during Q1 and Q2 of 2019 while the core program implementation commenced from Q3 2019 thereafter.

TB case finding activities were intensified during the grant through OPD screening strategy and free chest X-ray services for children. Designated staff referred to as screening officers were engaged to ensure $100 \%$ clinical TB screening of patient at the facility OPD thus promoting more presumptive TB yield from the private facilities engaged. The grant also supported both the cost of chest X-ray and the transportation to and from the X-ray facilities for children suspected of having TB in order to increase the number of childhood TB reported from the engaged private health facilities.

Sputum sample transportation to the GeneXpert sites was also strengthened with the use of paid motorcyclists and tracked with a web-based sputum dashboard. This was in a bid to increase DST coverage of all bacteriologically confirmed cases and promote sputum accountability.

\subsection{Data Collection}

Data was extracted from the routine registers: presumptive register; treatment register of the private health facilities engaged for PPM activities between 2019 and 2020. In addition to this, the data from the implementing states were ex- 
tracted at Local Government level from the central registers of LGTBLS. The data was collected using Microsoft excel package.

\subsection{Study Population}

The study population comprised of all presumptive TB patients who presented at the private health facilities engaged for the NFM2 programme between January 1, 2019 and December 31, 2020. For the purpose of this study, a patient is said to have been diagnosed as having tuberculosis if: he/she shows evidence of bacteriological confirmation with positive sputum AFB microscopy or Xpert MTB/ RIF test; he/she is diagnosed with clinical/radiological/histopathological confirmation irrespective of the outcome of the bacteriological test.

\subsection{Data Validation and Analysis}

In order to ensure validity of the data, only the data that were harmonized with those of the various State Tuberculosis and Leprosy Control Program Team members and LGTBLS were used in data analysis. Data was collected and checked for completeness and any error was corrected immediately. The data was entered and analyzed using Microsoft excel package. The variables were summarized using appropriate chart and table.

\section{Results}

Figure 2 below shows the quarterly contributions of the Global Fund Private Public Mix (GF PPM) to the total state TB notification in the four (4) states in South West Nigeria where the Global Fund NFM 2 grant was implemented. Overall, all the four states showed some progressive improvement in the GF PPM contribution to the total state TB notification, however at different rates. However, the percentage contributions of all the four states decreased between Q3

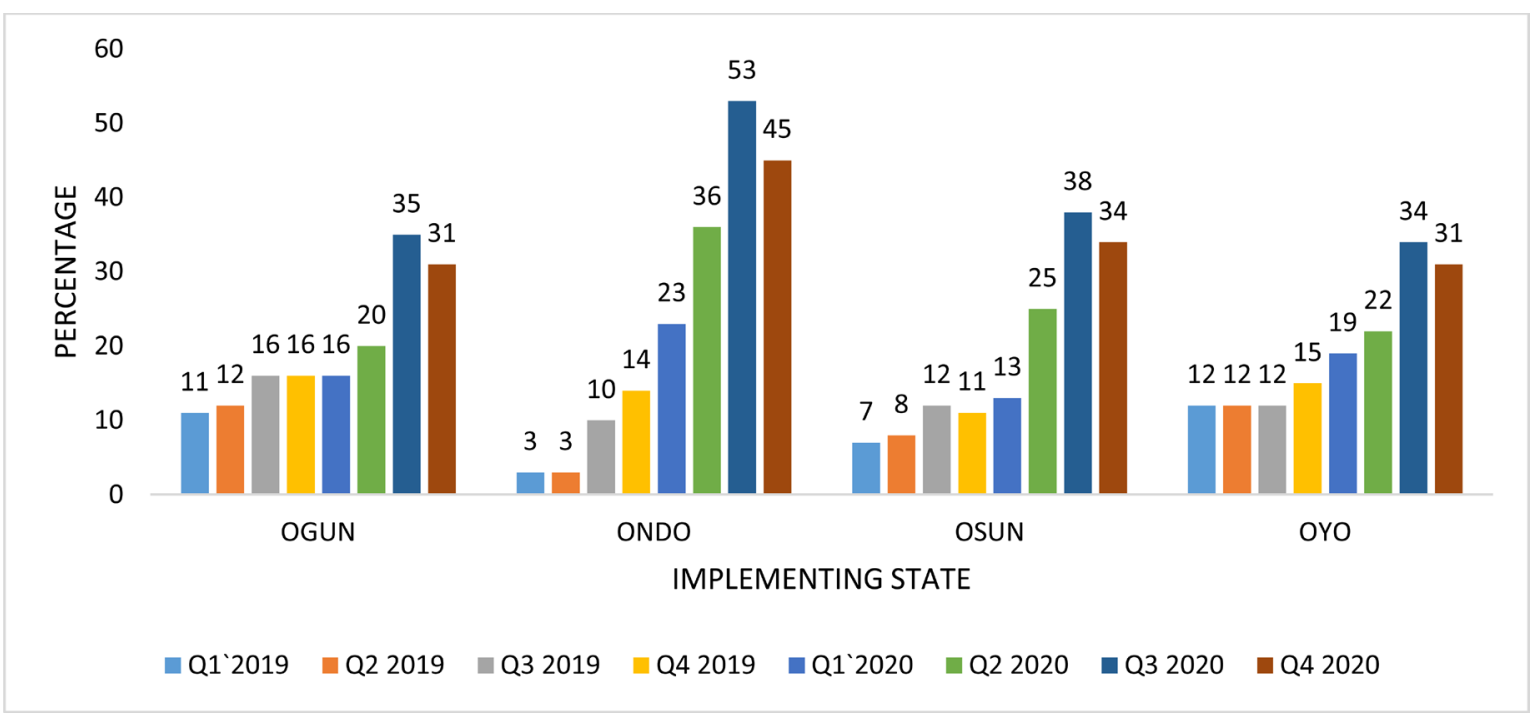

Figure 2. Quarterly contributions of GF PPM to total state TB notification on four states in South West Nigeria: Q1, 2019-Q3, 2020. 


\section{0 and Q4 2020.}

In Ogun state, the percentage contribution to PPM was $11 \%$ as at the first quarter of 2020. This however showed a marginal increase from $11 \%$ to $12 \%$ as the second quarter of the same year. The GF PPM contribution in Ogun State was however static at 16\% between Q3, 2019 and Q1, 2020. By Q3, 2020, the percentage contribution of GF PPM in Ogun State had increased to 35\%.

In Ondo state however, the percentage contribution of GF PPM to the state TB notification by Q1, 2019 was only 3\%. This increased progressively and by Q3 2020, 53\% of all the notified TB cases Ondo State were from GF PPM. By Q4 2020, the percentage contribution of GF PPM to the total state TB notification in Ondo State had decreased to $45 \%$.

At Q1, 2019, the percentage contribution of GF PPM to Osun and Oyo States TB notification were $7 \%$ and $12 \%$ respectively. By Q3, 2020, the percentage contribution of GF PPM to these two states had risen to $38 \%$ and $34 \%$ respectively.

Figure 3 below shows the quarterly trend of presumptive TB case generation among all the four GF PPM implementing states in south west Nigeria between Q1, 2019 and Q4, 2020. As depicted in the figure, 707 presumptive TB cases were generated in Q1, 2019. The number of presumptive TB cases generated in Q2, 2020 decreased to 504 presumptive TB cases. Thereon, this number increased progressively and the number of presumptive TB cases had increased to17,836 cases at the end of Q4 2020.

Figure 4 below shows the percentage contribution of the different providers to the presumptive TB cases generated between Q1, 2019 and Q4, 2020. In Q1, $2019,100 \%$ of the presumptive TB cases were generated by the health facilities. However, in Q2, 2019, 96\% of the presumptive TB cases were generated by the health facilities, while only $3 \%$ of the presumptive TB cases were generated by the PPMVs. By Q4 2020, the health facilities were responsible for about 32\% of the total presumptive TB cases generated while the PPMVs were responsible for about $68 \%$ of the presumptive TB cases generated. Furthermore, all through the period under review, no presumptive TB case was ascribed to the community

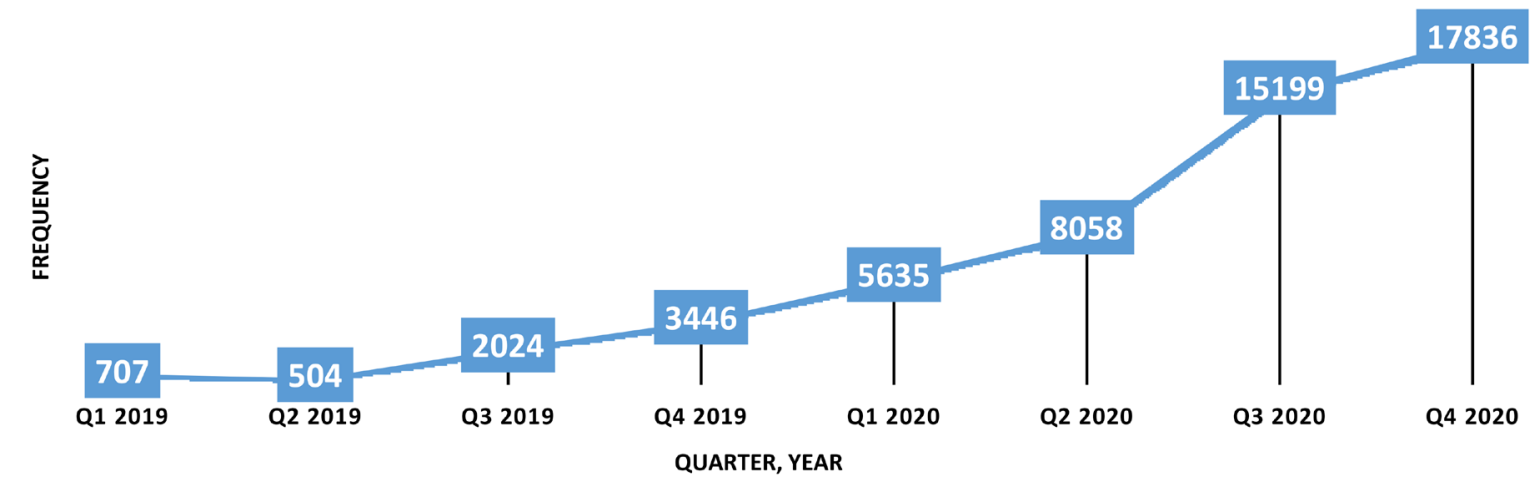

Figure 3. Quarterly trend of presumptive TB cases generated Q1 2019-Q4 2020. 
pharmacists as well as the stand-alone laboratories.

Table 1 below shows the quarterly distribution of the diagnosed TB cases and the percentage of TB cases notified by implementing states. Overall and during the period under review, majority of the implementing states revealed some progressive increase in the proportion of diagnosed TB cases that were notified. In Q1 2019, the proportion of diagnosed TB cases who were notified were $0 \%$ in Ogun State; $34.8 \%$ in Osun State; $65.9 \%$ in Oyo State; and $85.7 \%$ in Ondo State. By Q4 2020, the proportion of TB cases who were notified include: $88.3 \%$ in Ogun State; $95.5 \%$ in Ondo State; $99.3 \%$ in Osun State; and 100\% in Oyo State.

\section{Discussion}

This study set out to evaluate the contribution of the private health sector to the tuberculosis notification in four selected states in South West Nigeria between 2019 and 2020. In this study, it was reported that there was a progressive increase in the presumptive $\mathrm{TB}$ cases attributable to the efforts of the private health

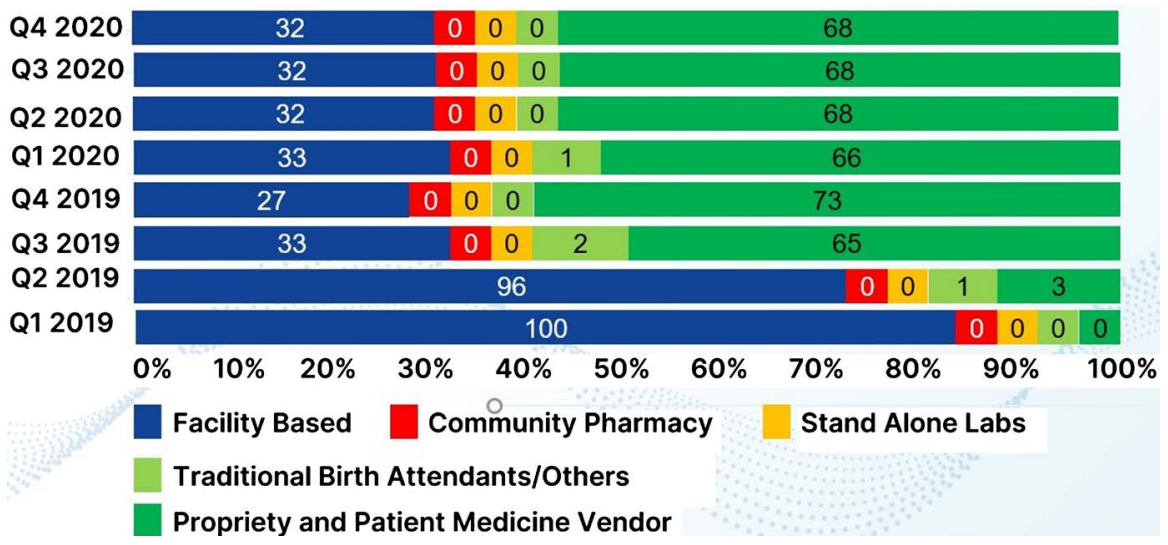

Figure 4. Percentage contributions of private providers to total presumptive TB cases generated in the four implementing states: Q1 2019-Q4 2020.

Table 1. Percentage TB notification amongst the four implementing states in South West Nigeria: Q1 $2019-\mathrm{Q} 42020$.

\begin{tabular}{|c|c|c|c|c|c|c|c|c|c|c|c|c|c|c|c|}
\hline \multirow{2}{*}{$\begin{array}{l}\text { STATE } \\
\text { Quarter }\end{array}$} & \multicolumn{3}{|c|}{ OGUN } & \multicolumn{3}{|c|}{ ONDO } & \multicolumn{3}{|c|}{ OSUN } & \multicolumn{3}{|c|}{ OYO } & \multicolumn{3}{|c|}{ TOTAL } \\
\hline & 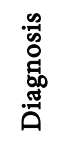 & 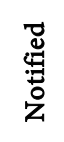 & 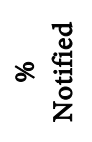 & 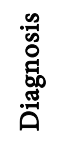 & 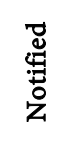 & 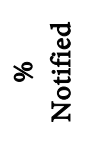 & 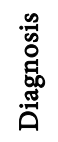 & 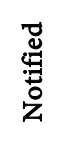 & ஃ。 & $\begin{array}{l}\stackrel{9}{0} \\
0 \\
\stackrel{0}{0} \\
\stackrel{\Xi}{0} \\
\ddot{0}\end{array}$ & 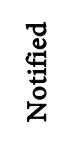 & ๙ & 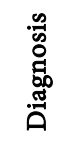 & 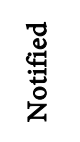 & $\therefore$ 苞 \\
\hline Q1 2019 & 5 & 0 & 0.0 & 7 & 6 & 85.7 & 43 & 15 & 34.8 & 88 & 58 & 65.9 & 143 & 79 & 55.2 \\
\hline Q2 2019 & 35 & 5 & 14.3 & 9 & 6 & 66.7 & 33 & 12 & 36.3 & 69 & 49 & 71.0 & 146 & 72 & 49.3 \\
\hline Q3 2019 & 120 & 16 & 13.3 & 40 & 40 & 100.0 & 115 & 98 & 85.2 & 106 & 100 & 94.3 & 381 & 254 & 66.7 \\
\hline Q4 2019 & 118 & 47 & 39.8 & 53 & 53 & 100.0 & 108 & 105 & 97.2 & 179 & 175 & 97.7 & 458 & 380 & 83.0 \\
\hline Q1 2020 & 136 & 58 & 42.6 & 99 & 97 & 98.9 & 122 & 110 & 90.1 & 229 & 219 & 95.6 & 586 & 484 & 82.6 \\
\hline Q2 2020 & 105 & 47 & 44.7 & 116 & 110 & 94.8 & 262 & 243 & 92.7 & 266 & 250 & 93.9 & 749 & 650 & 86.8 \\
\hline Q3 2020 & 155 & 119 & 76.7 & 203 & 191 & 94.0 & 669 & 657 & 98.2 & 599 & 599 & 100.0 & 1626 & 1566 & 96.3 \\
\hline Q4 2020 & 154 & 136 & 88.3 & 203 & 194 & 95.5 & 786 & 781 & 99.3 & 576 & 576 & 100.0 & 1719 & 1687 & 98.1 \\
\hline
\end{tabular}


providers engaged in the GF PPM NFM2 grant. It was also reported that with the 2-year grant, the percentage contribution of the private healthcare providers rose in the course of the implementation of the grant.

Nigeria is a country with a very weak health system; poor health seeking behavior as well as a vast but largely unregulated private health sector. In addition to this, due to the non-homogenous nature of the private health providers especially in low resource settings such as Nigeria, there is still a lot to be known and reported about this sector [8]. In addition, one strategy/approach does not fit the programmatic engagement of all the members of the private health providers [8]. Due to the non-homogenous nature of these private providers, the services rendered by this sector are often sub-standard in nature. However, non-engagement of this large sector could have its consequences on the control of tuberculosis especially in a low resource country such as Nigeria [9]. The consequences could include: increase in transmission as a result of delay in diagnosis and treatment of this infectious disease; increase in morbidity and mortality owing to the substandard nature of the treatment; increase in the drug resistant variant of the disease due to sub-therapeutic dosages; cost of treatment could be catastrophic because the private health providers are in business to make money; and there will be incomplete data reportage and thus the real burden of the disease will be unknown [9]. The private sector provides up to $60 \%$ of the total healthcare delivery In Nigeria [10]. The private health sector in Nigeria is diverse and heterogeneous, comprising of the orthodox and the traditional settings which have little or no connections or collaboration [11]. These private facilities are largely unregulated and are often bedeviled by weak referral systems [10]. It is pertinent to engage the private facilities in the diagnosis and management of tuberculosis.

During the NFM2 funding cycle, there was a progressive increase in the number of presumptive TB cases generated through the engagement of the private sector. OPD screening was probably one of the success stories of the presumptive TB generation drive during the period under review. Screening officers were engaged and deployed to private health facilities to screen patients verbally for tuberculosis using its symptom case definition. In order to ensure that screening of OPD attendees is done effectively, the Mobile Application for Tuberculosis Screening (MATS) application was deployed for this purpose. This is a mobile application which works with Android phones for the purpose of screening of OPD attendees and other patients. This has an advantage over the cumbersome nature of the paper-based screening methods as screening by MATS application was faster and reduce the delays involved in decision making process and care of the patients. Those who met the case definition of tuberculosis were counselled and made to undergo bacteriological testing. Furthermore, incentivizing the diagnosis of the presumptive TB cases tested through the hubs as well as the spokes also contributed to the increase in the number of presumptive cases being generated by the private providers. As reported in a previous study, paying incentives for presumptive case generation might lead to unnecessary bacteriological 
testing by the private providers whose sole aim of providing health care to the patients is to maximize profit [12]. In order to mitigate this, incentives are paid with the proviso that at least one TB case must be detected in 10 presumptive TB cases generated. Other strategies implemented to increase the drive for presumptive TB generation were: demand creation for TB services; mandatory contact racing for all new cases of TB detected; as well as community outreaches in communities suspected with a high burden of the disease [13] [14].

At the beginning of the NFM2 grant, the health facilities contributed virtually all of the presumptive TB cases in the four states. However, as the programme progressed, more presumptive TB cases were coming from the patent medicine vendors than the private health facilities. It was also noted that other providers such as the community pharmacists; stand-alone laboratories; the traditional birth attendants and other private providers contributed little or nothing to the presumptive TB case generation drive in the four south western states. This shows the heterogeneous nature of the private providers. The reason for this is not farfetched. As noted in previous studies, one of the challenges of the private provider engagement for TB services is the heterogeneous nature of the private providers, and as such, bringing them together under one single program is a herculean task [15] [16] [17]. Moreover, some subtle rivalry was noted between the PPMVs and the community pharmacists. This affected the level of engagement of the community pharmacists. As compared to the PPMVs, a relative reduction in the number of presumptive TB cases generated in the health facilities could be due to the burden resulting from their dual role of tuberculosis case finding and management as hubs to the various spokes in the hub and spoke model. Furthermore, based on the available data on the contribution of the various private providers to the generation of presumptive TB cases, the private providers are far from being saturated. Thus, it is clear that there are lots of opportunities for a scale up of private provider engagement in the selected states in South West Nigeria. Due to the heterogeneous nature of the private providers, one of the ways to scale up on the level of engagement of the private providers is through a more robust discussion with the various association bodies of the private providers on an individual basis and not collectively.

Most of the selected states in the NFM2 grant showed progressive increase in the contribution of the private providers to tuberculosis notification. At the beginning of the NFM2 funding cycle, proportion of the notified TB cases from the private providers was abysmally low and called for a lot of concern. However, as the funding cycle progressed, the contributions of the private providers to TB case notification increased. Even at the end of the cycle, the private providers in Ondo state were responsible for more than half of all the notified TB cases in the state. According to the 2015-2020 national strategic plan on tuberculosis control by the National Tuberculosis and Leprosy Control Program (NTBLCP), private providers were expected to contribute about $30 \%$ of the tuberculosis case notification [10]. At the sub-national level, available data has shown that this feat was 
surpassed by most of the selected states as reported in this study. Some of the innovative strategies deployed to increase the tuberculosis case notification by the private entities were regular supportive supervisory visits to the private health providers; effective tracking of the samples from the points of sample collection to the GeneXpert/AFB microscopy sites using a web-based application; incentivizing case notification; as well as effective monitoring and evaluation of activities. As noted in other studies, effective collaboration with the private providers can increase the TB case notification if well harnessed [12] [18] [19].

This study is not without its own limitations. First, the study is an operational research which focused only on the engagement of private providers in the four implementing states of Damien Foundation as a sub-recipient to the Institute of Human Virology, Nigeria. As such, these states were not selected using any probability sampling technique. Thus, generalization of its findings might not be possible. Secondly, this research team is not privy to data on the number of patients who refused OPD screening as well as data on private providers who could have provided TB diagnostic and treatment services, however informal it might have been, but were not formally engaged on the PPM grant. In spite of the above limitations, this study has been able to showcase the contributions of the private providers to the diagnosis and management of tuberculosis across the south western states of Nigeria involved in the NFM2 grant.

\section{Conclusion}

Though heterogeneous and largely unregulated, private providers are an important entity in the Nigerian health system. They constitute a large part of the Nigerian health system, and payments for their services are often out-of-pocket and could be catastrophic in nature. As a fall out of these, this study evaluated the contributions of the private provider engagement in the four implementing states of the south west Nigeria to the total tuberculosis case finding and notification. With an increase in private provider engagement in these states in the NFM2 funding cycle, there is a progressive increase in the number of presumptive TB cases as well as in the number of notified cases of tuberculosis. Furthermore, the contribution of the private providers to the tuberculosis case notification in most of the states is progressively increasing. Moreover, the PPMVs tend to contribute more to the overall number of presumptive TB cases than any other cadre of private providers. Thus, there are still opportunities for a scale up in the private provider engagement in these states.

\section{Conflicts of Interest}

The authors declare no conflicts of interest regarding the publication of this paper.

\section{References}

[1] World Health Organization (2020) Global Tuberculosis Report 2020. World Health Organization, Geneva. 
[2] World Health Organization (2015) The End TB Strategy. World Health Organization, Geneva.

[3] Stallworthy, G., Dias, H.M. and Pai, M. (2020) Quality of Tuberculosis Care in the Private Health Sector. Journal of Clinical Tuberculosis and Other Mycobacterial Diseases, 20, Article ID: 100171. https://doi.org/10.1016/j.jctube.2020.100171

[4] Welcome, M. (2011) The Nigerian Health Care System: Need for Integrating Adequate Medical Intelligence and Surveillance Systems. Journal of Pharmacy \& Bioallied Sciences, 3, 470-478. https://doi.org/10.4103/0975-7406.90100

[5] Stallworthy, G., Wells, W. and Dias, H.M. (2018) Engaging Private Health Care Providers in TB Care and Prevention: A landscape Analysis. World Health Organization, Geneva.

[6] Ukwaja, K.N., Alobu, I., Nweke, C.O. and Onyenwe, E.C. (2013) Healthcare-Seeking Behavior, Treatment Delays and Its Determinants among Pulmonary Tuberculosis Patients in Rural Nigeria: A Cross-Sectional Study. BMC Health Services Research, 13, Article No. 25. https://doi.org/10.1186/1472-6963-13-25

[7] Konduri, N., Delmotte, E. and Rutta, E. (2017) Engagement of the Private Pharmaceutical Sector for TB Control: Rhetoric or Reality? Journal of Pharmaceutical Policy and Practice, 10, Article No. 6. https://doi.org/10.1186/s40545-016-0093-3

[8] Khan, M.S., Salve, S. and Porter, J.D.H. (2015) Engaging for-Profit Providers in TB Control: Lessons Learnt from Initiatives in South Asia. Health Policy and Planning, 30, 1289-1295. https://doi.org/10.1093/heapol/czu137

[9] World Health Organization (2018) Public-Private Mix for Tb Prevention and Care: A Roadmap. World Health Organization, Geneva.

[10] NTBLCP (2014) The National Strategic Plan for Tuberculosis Control, 2015-2020: Towards Universal Access to Prevention, Diagnosis and Treatment. National TB and Leprosy Control Programme, Abuja.

[11] NTBLCP (2021) Human Rights and Gender Action Plan for Tuberculosis Care and Prevention in Nigeria (2021-2025). National TB and Leprosy Control Programme, Abuja.

[12] Anand, T., Babu, R., Jacob, A.., Sagili, K. and Chadha, S. (2017) Enhancing the Role of Private Practitioners in Tuberculosis Prevention and Care Activities in India. Lung India: Official Organ of Indian Chest Society, 34, 538.

https://doi.org/10.4103/0970-2113.217577

[13] Lorent, N., Choun, K., Thai, S., Kim, T., Huy, S., Pe, R., et al. (2014) CommunityBased Active Tuberculosis Case Finding in Poor Urban Settlements of Phnom Penh, Cambodia: A Feasible and Effective strategy. PLoS ONE, 9, e92754.

https://doi.org/10.1371/journal.pone.0092754

[14] Singh, D., Negin, J., Otim, M., Orach, C.G. and Cumming, R. (2015) The Effect of Payment and Incentives on Motivation and Focus of Community Health Workers: Five Case Studies from Low- and Middle-Income Countries. Human Resources for Health, Human Resources for Health, 13, Article No. 58. https://doi.org/10.1186/s12960-015-0051-1

[15] Naser, A., Ullah, Z., Huque, R., Husain, A., Akter, S., Islam, A., et al. (2012) Effectiveness of Involving the Private Medical Sector in the National TB Control Programme in Bangladesh : Evidence from Mixed Methods. BMJ Open, 12, Article ID: e001534. https://doi.org/10.1136/bmjopen-2012-001534

[16] Deo, S., Singh, S., Jha, N., Arinaminpathy, N. and Dewan, P. (2020) Predicting the Impact of Patient and Private Provider Behavior on Diagnostic Delay for Pulmonary Tuberculosis Patients in India: A Simulation Modeling Study. PLoS Medicine, 
17, e1003039. https://doi.org/10.1371/journal.pmed.1003039

[17] Deo, S., Singh, S., Khaparde, S., Rao, R., Vadera, B., Kulshrestha, N., et al. (2018) Delays, Behaviour and Transmission: Modelling the Impact of Effective Private Provider Engagement on Tuberculosis Control in Urban India. BioRxiv, Article ID: 461426.

[18] Vo, L.N.Q., Codlin, A.J., Huynh, H.B., Mai, T.D.T., Forse, R.J., Van Truong, V., et al. (2020) Enhanced Private Sector Engagement for Tuberculosis Diagnosis and Reporting through an Intermediary Agency in Ho Chi Minh City, Viet Nam. Tropical Medicine and Infectious Disease, 5, 143.

https://doi.org/10.3390/tropicalmed5030143

[19] Ananthakrishnan, R., D’Arcy Richardson, M., Van Den Hof, S., Rangaswamy, R., Thiagesan, R., Auguesteen, S. et al. (2019) Successfully Engaging Private Providers to Improve Diagnosis, Notification, and Treatment of TB and Drug-Resistant TB: The EQUIP Public-Private Model in Chennai, India. Global Health Science and Practice, 7, 41-53. https://doi.org/10.9745/GHSP-D-18-00318 